\title{
A $d q$ axis theory of the magnetic, thermal, and mechanical properties of Curie motor
}

\author{
Marco Trapanese ${ }^{a)}$ \\ Dipartimento di Ingegneria Elettrica, Elettronica e delle Telecomunicazioni, Palermo University, \\ Palermo, I-90128 Palermo, Italy
}

(Presented 18 November 2010; received 4 October 2010; accepted 1 December 2010; published online 00 April 2011)

\begin{abstract}
A $d q$ axis theory of a thermomagnetic Curie motor is presented. This theory allows one to estimate the performances of a Curie motor from its geometrical, magnetic, and thermal properties. The proposed approach shows that the thermomagnetic Curie motor is equivalent from a magnetic point of view to a dc electric machine. The physical meaning of the parameters used in the $d q$ theory of Curie motor is explicated. The theory is validated by using experimental data.
\end{abstract} (C) 2011 American Institute of Physics. [doi:10.1063/1.3562505]

\section{INTRODUCTION}

Direct conversion of thermal energy into kinetic energy can be obtained in a Curie motor. ${ }^{1,2}$ This type of motor consists of a magnetic circuit with a soft magnetic rotor in its gap and no electric current is involved in the force generation process because the force is generated by a thermally induced permeability difference of two areas of the rotor. More precisely, the heating of one side of the rotor modifies locally the permeability of the soft magnetic material in comparison with the cool area of the rotor and this permeability difference causes a force. If the temperature difference is continuously kept, a continuous movement of the rotor is obtained.

The feasibility of the motor has been shown several times, ${ }^{3}$ but in all the experimental applications a very low speed and a relatively low torque have been obtained. This is caused by the fact that in the force generation process two physical phenomena (thermalization and magnetization) characterized by two different time scales are involved.

This paper presents an approach to the description of the Curie motor that is based on the $d q$ axis general theory of electric machines. The stator is modeled by assuming the use of a high performance permanent magnet. The flux and its temperature are supposed to be constant. The rotor is modeled in terms of both its magnetic as well thermal properties. It is supposed to have a temperature gradient between the hot and cold side. This model provides analytical expressions for the speed and torque that link these quantities to some parameters which synthetically describe the physical phenomena involved in the principle of operation of the motor (thermalization and magnetization). The expressions of speed and torque are derived and related to the thermal properties of the machine. The dependence from these fixed parameters is also discussed and it is shown how a higher value of the magnetization field as well a higher value of the temperature gradient positively influence motor performances. An experimental verification of the performances is reported.

${ }^{\text {a)} E l e c t r o n i c ~ m a i l: ~ m t r a p @ d i e p a . u n i p a . i t . ~}$

\section{THE PRINCIPLE OF OPERATION OF A CURIE MOTOR}

The principle of operation of the Curie-motor can be outlined as in Fig. 1. For the sake of simplicity, a Curie linear motor is considered; it consists of a soft magnetic, movable armature and a fixed field source. The magnetic flux is generated by permanent magnet. A homogeneous magnetic field without leakage is assumed. To operate the motor, one side of the armature is heated. Because of heat conduction along the armature, a temperature gradient arises and the magnetic properties along the armature change. The cold side of the rotor is kept at temperature by cooling. If the warm side of the armature is heated above Curie temperature, it behaves there magnetically like air or vacuum. As a result, a permeability difference is generated and a force arises in the direction of the warm side. If the armature is movable, it is drawn into this direction. Under the mentioned conditions the $\mathrm{Cu}$ rie-motor performs like a conventional magnetic device. In reality, however, the Curie-motor will not produce a sharp boundary surface between the warm and cold side.

\section{III. dq AXIS THEORY OF CURIE MOTOR}

Generally speaking, $d q$ axis theory of electromagnetic machines ${ }^{4}$ could be used to describe any electromagnetic machine. However, in the case of the Curie motor there is a fundamental difficulty: in the Curie motor neither currents nor voltages are involved. So, superficially, one could think that Park theory is not applicable to Curie motor. In fact, this difficulty can be solved by reasoning as outlined in the following.

In Fig. 2 it is shown how the field distribution shown in Fig. 1 can be obtained by placing a magnet in quadrature with the excitation magnets. The magnetic structure shown is the magnetic structure of a dc electrical machine where the fictious magnet that describes the effect of the thermal field plays the role of the magnets generated inside the armature of a dc machine. As a result, from an electromagnetic point of view a Curie motor can be described by two constant magnets placed in quadrature and therefore it is 


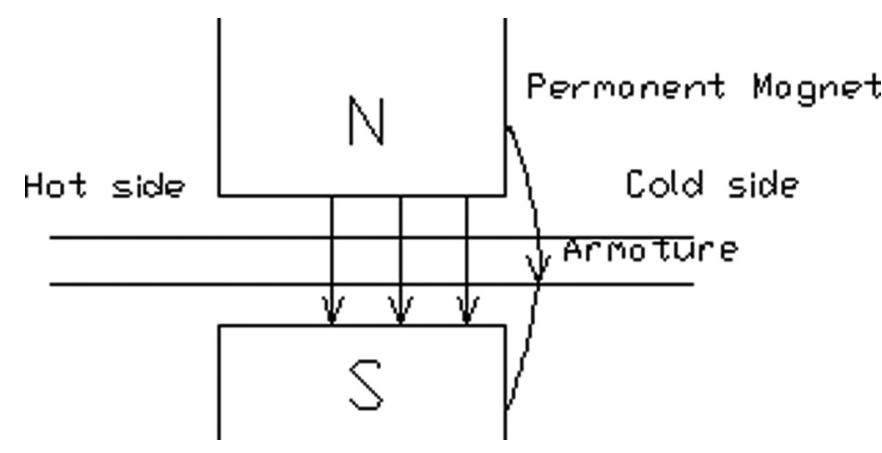

FIG. 1. Field distribution when the temperature is not uniform along the armature. The field distortion caused by the temperature difference that induces a permeability difference generates a force.

equivalent to a dc machine. Therefore, the equations used for the description of a dc machine can be used to describe a $\mathrm{Cu}-$ rie motor.

According to what was said earlier in this paper, the set of dynamic equations that describe a Curie motor, under the hypothesis of a constant excitation field, reads as follows:

$$
\begin{gathered}
\phi=k I_{d}=\mathrm{const}, \\
C=M_{d q} I_{d} I_{q}, \\
V_{q}=R_{q} I_{q}+\omega M_{d q} I_{d},
\end{gathered}
$$

where $\varphi$ is the excitation flux, $k$ is a constant, $I_{d}$ is a current in correspondence of the direct axis that is able to generate the excitation flux, $V_{q}$ is the voltage on the quadrature axis circuit, $R_{q}$ is the resistance of the armature, $I_{q}$ is a current in correspondence of the quadrature axis that describes the magnetic poles needed to generate the field distribution induced by the temperature gradient, $\omega$ is the rotational speed, $M_{d q}$ describes the magnetic coupling between $d$ and $q$ axes, and $C$ is the electromagnetic torque. Due to the fact that no electrical circuit is located on the moving part of a real Curie motor, Eqs. (1b) and 1(c) raise the following question: which is the physical meaning of the electrical quantities used in those equations?

\section{PHYSICAL MEANING OF $d q$ EQUATIONS}

Equation (1a) describes the excitation flux. Due to the fact that excitation is made of a permanent magnet, Eq. (1) is absolutely identical to the equation used to describe the excitation of a traditional dc machine. As a result $I_{q}$ is a current

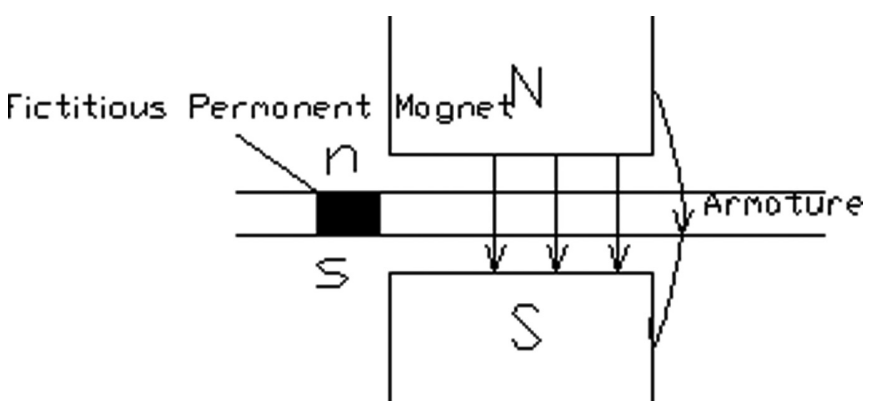

FIG. 2. The field distribution of Fig. 1 can be reproduced by adding a magnet when the temperature is uniform. that describes the excitation flux and, in a first approximation, is constant and does not depend on the armature temperature.

Equation (1b) describes the torque generated by the $\mathrm{Cu}-$ rie motor and contains the novelties related to the description of a Curie motor. In a Curie motor the torque is generated by the permeability difference between the hot and cold spots. As already stated, $I_{d}$ describes the excitation and, therefore, $I_{q}$ describes the permeability difference induced by the temperature gradient. At standstill $T_{h}$ and $T_{c}$ are governed by the convection heat exchange with the cooling and heating system and the thermal conduction between the hot and cold spots inside the rotor. In order to have an accurate description of this phenomenon, one should use a numerical approach, but in order to grasp the physics of the phenomenon it is better to use the following analytical equation at lumped parameters to express the temperature difference:

$$
T_{c}-T_{h}=\frac{L}{k S} \cdot \frac{d q}{d t},
$$

where $T_{c}$ is the temperature at the cold spot, $T_{h}$ is the temperature at hot spot, $L$ is the distance, $k$ is the thermal conductivity, $S$ is the surface, and $d q / d t$ the heat rate. At standstill, heat flows from the hot spot to the cold spot and a constant temperature gradient is established. The maximum and minimum temperature $T_{h}$ and $T_{c}$ can be adjusted by adjusting the heat rate that is imposed by the temperature of the cooling and heating fluids. This temperature imposes (through the magnetic characteristics of the material) the permeability difference and therefore determines $I_{q}$. If one assumes that $T_{h}$ and $T_{c}$ are, respectively, above and below the Curie temperature of the material and that permeability difference around the ferromagnetic critical point is proportional to a power of this temperature difference, one can express $I_{q}$ as follows:

$$
I_{q}=k_{1} \Delta T^{v}=k_{1}\left(\frac{L}{k S} \frac{d q}{d t}\right)^{v},
$$

where $v$ is the exponent that describes the dependence of the permeability difference on the temperature difference, and $k_{1}$ is a constant that allows one to match the lumped parameter to the real values. If Eq. (3) is compared to Eq. (1c), one can see that voltage in $d q$ theory describes the heat rate (in a nonlinear way if $v$ is different from 1). obtains:

If Eq. (3) is used to express in $I_{q}$ in Eq. (1b), one

$$
C=\frac{M_{d q} k_{1} L^{v}}{k^{v} S^{v}} I_{d} \cdot\left(\frac{d q}{d t}\right)^{v} .
$$

If the rotor is moving the physical phenomena related to heat exchange do not remain the same but one must also include the advection (the change in the thermal energy of a mass as it moves through space). As a result, one can show that the temperature difference can be roughly expressed (at least in the regimes feasible for a Curie motor) as

$$
\Delta T=k_{2} e^{-d \omega}
$$

where $\mathrm{d}$ is the distance between hot and cold spots and $\omega$ is the angular speed, $k_{2}$ is an angular constant that takes into 
Angular speed vs time

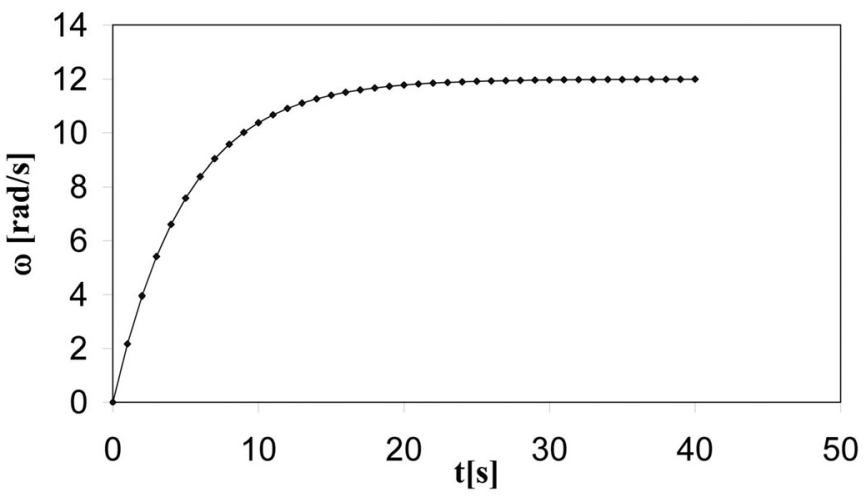

FIG. 3. Transient behavior of a disk-shaped Curie rotor.

account the geometrical details. First-order approximation of Eq. (5) is

$$
\Delta T=k_{2}(1-d \omega)
$$

Equation (6) shows that advection causes a reduction of the generated temperature difference [the last term in Eq. (6)] and consequently of $I_{q}$ and of the generated torque. Formally speaking, advection plays the role of the back electromotive force term in Eq. (1c).

\section{EXPERIMENTAL VALIDATION}

By using the above-described approach, some experimental results can be explained. This section refers to a diskshaped superconducting Curie rotor levitated between two magnets that guarantee the levitation force and the excitation of the rotor. The numerical data and the experimental results are published in Ref. 2 and therefore are not repeated here. By assuming that $v$ is equal to one and by using Eq. (6), the equation of motion of rotor in this case reads as

$$
M_{d q} I_{d} k_{2}(1-d \omega)=I \frac{d \omega}{d t}
$$

where $I$ is the inertia of the rotor. By using the numerical data given in Ref. 2, Eq. (7) can be solved and the time evolution is shown in Fig. 3. If one compares this solution with the results published in Ref. 2, one can see that the behavior of the angular speed is correctly described from the theory presented here and the transient time scale derived is of the order of $10 \mathrm{~s}$ as is the one in Ref. 2. However, the oscillations in the angular speed that are shown in Ref. 2 are not described in the theory presented here because they are caused from a coupling between the suspension technique of the rotor (levitation in Ref. 2) and its rotational movement. This coupling is not included in the theory here presented because the position of the rotation center is supposed constant.

\section{CONCLUSIONS}

In this paper a $d q$ theory of a Curie motor has been presented. The theory is able to link the electrical parameters of $d q$ theory to the physical phenomena occurring in a Curie motor. It is shown how the temperature difference plays a fundamental role for torque generation and that the temperature difference is governed by both thermal conduction as well as advection in the rotor. Finally, an experimental validation of the theory has been presented.

\section{ACKNOWLEDGMENTS}

This work was supported by MIUR (project "Finanziamenti Ricerca Scientifica di Ateneo 2005" ex quota 60\%).

${ }^{1}$ N. Tesla, U.S. Patent 396,121 (1889).

${ }^{2}$ C. Palm, Europhys. News 38, 32 (2007).

${ }^{3}$ K. Murakami and M. Nemoto, IEEE Trans Magn. 8, 387 (1972).

${ }^{4}$ R. H. Park, AIEE Trans. 48, 716 (1929). 
AQ1: For consistency purposes, $d q$ is used throughout the text. Please check.

AQ2: Please shorten your paper so that it fits within the 3 page length limit for MMM contributed papers.

AQ3: Please check edits to the sentence that begins "This section refers to a disk-shaped..."

AQ4: Please provide DOI for the Ref. 1, 4. 